\title{
Improved RFID Middleware Architecture and Optimal Algorithm based on Internet of Things
}

\author{
Weiqing Qu, Yuedou Qi, Qi Zhang and Chunliang Zhou \\ Ningbo Dahongying University, Ningbo 315175, China \\ *qwq606@aliyun.com.
}

\begin{abstract}
Internet of things is a wireless data communications technology, while radio frequency identification (RFID) is an emerging technology in the automatic identification of the Internet of things. In fact, it means the use of RFID system identification technology through the Internet to achieve the application of information interconnection and sharing. In this paper, the author analyzes an improved RFID middleware architecture and optimal algorithm based on internet of things. Through the analysis of RFID middleware technology, we put forward an optimized load balancing algorithm. The results show that the optimization algorithm is superior to the existing algorithms in the average time of load balancing and label processing.
\end{abstract}

Keywords: RFID middleware, Optimal algorithm, Internet of things, Load balancing mechanism

\section{Introduction}

The Internet means the use of computer network in the wireless data communication technology and frame covering all global Internets, and radio frequency identification RFID (Radio Frequency Identification), is a new automatic identification technology in the Internet of things[1]. In this network, the object and the object can begin to communicate by the automatic intelligent identification technology, but not by human intervention[2-3]. In fact, the use of RFID system identification technology through the Internet to achieve the application of information interconnection and sharing. Internet of things to be embedded in the sensor to identify and be identified by the Internet in the integration of the Internet, to achieve effective integration of human society and the physical system[4]. And RFID technology is precisely to make things "speak" a recognition technology. RFID technology is a technology that uses radio waves to transmit information between an electronic tag and reader. It is created by a seamless connection between the physical object and the digital native.

The RFID system is attached to the object by the RFID tag identifier and automatically uses the wireless communication to make a single object unique. Compared with traditional identification techniques, such as magnetic stripe or bar code, RFID refers to the line of sight without limit, a non-contact technique[5-6]. Although the RFID system is based on the underlying technology of the surrounding infrastructure, all modern RFID system can be divided into three main parts, namely, tag, reader and back-end systems. There is in today's world, the research of RFID middleware is also in an important period of time, with the development of networking and cloud computing has been a breakthrough in the primary stage, and in the Internet plays an important core technology RFID technology also with its research and development and the emergence of many practical problems[7-8]. In these problems, related to the load balancing problem of cross module RFID middleware and cloud computing is the middleware, due to the use of this kind of recognition technology widely, and allows the system to integrate a single frame of a huge networking center in this kind of system, but also as the basis of cloud 
computing technology[9]. The system has many difficult problems, such as radio frequency identification technology of our daily common is the two generation ID card, and the two generation ID card system of the Ministry of public security in the past is an independent system, and other related systems without cross or less. But with the social and economic development, technical innovation and breakthroughs develop.

\section{RFID Technology Research}

\subsection{RFID}

At present, the research focus on the RFID technology is the frequency, antenna design, anti collision and security technology of the hardware manufacturers in foreign countries. One of the key problems in RFID technology is the choice of operating frequency, there are many factors to be considered in the selection of working frequency, such as a variety of different applications, and all of the radio frequency to use and emission limits and scope. And the frequency of RFID is across multiple frequency bands, but the different frequency bands have their advantages and disadvantages. Low frequency wireless coverage without high frequency wireless coverage, but due to the diffraction of low-frequency Youbo, which can bypass the barrier, but the low frequency electronic label costs relatively low, a card shaped, strip, ring and so on are shown in Table 1 is characteristic of low frequency and high frequency.

\section{Table 1. RFID Frequency Band Characteristics Statistical Analysis of the Questionnaire}

\begin{tabular}{|c|c|c|c|}
\hline Band & Describe & Action distance & Penetration ability \\
\hline $125-134 \mathrm{KHz}$ & Low frequency & $45 \mathrm{~cm}$ & $\begin{array}{c}\text { Can penetrate most } \\
\text { of the objects }\end{array}$ \\
\hline $13.553-13.567 \mathrm{MHz}$ & High frequency & $1-3 \mathrm{~m}$ & $\begin{array}{c}\text { More able to } \\
\text { penetrate the liquid } \\
\text { and metal }\end{array}$ \\
\hline $400-1000 \mathrm{MHz}$ & Ultra high frequency & $3-9 \mathrm{~m}$ & $\begin{array}{c}\text { Penetrating power is } \\
\text { weak }\end{array}$ \\
\hline $2.45 \mathrm{GHz}$ & Microwave & $3 \mathrm{~m}$ & Weakest penetration \\
\hline
\end{tabular}

Because there are multiple electronic tags in the same frequency range for the study of RFID security technology, access control mechanism so that the radio frequency signal will appear in the output signal without using multiple access errors, so using one or several algorithms to solve the conflict between multiple overlapping working range reader error, such as Colorware algorithm and classic ALOHA protocol can solve the conflict information output part. But these two algorithms have their advantages and disadvantages, and both belong to the time division multiple access algorithm. RFID based on technology is one of the key technologies in the Internet of things, so the scope of the application of RFID is wide, coupled with the application of the RFID technology is also constantly widely. Such as logistics and Internet combined application of RFID technology is hot, when the goods are loaded on each or RFID label, so things such RFID technology of manufacturing, marketing, logistics, use, reuse and other aspects of the circulation of goods plays a profound impact, there is the RFID books in the world any item can be connected to the Internet through the label identification, tracking, monitoring and management, the cost and time saving. Internet of things is following the IT Internet also has a major revolution. Although the Internet of things technology has not yet developed mature, but there is the prototype of the Internet of things is the RFID technology. 


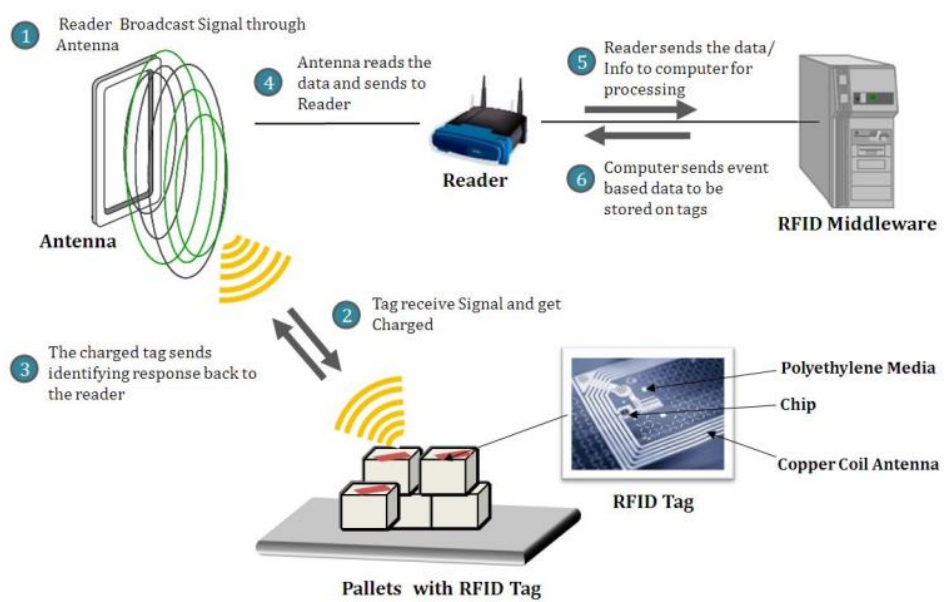

Figure 1. RFID Technology

Research on RFID technology, the first is the analysis and design of the RFID architecture, the RFID architecture is built on top of the middleware, a different reason of RFID middleware is the reader of the deployment is not the same, which can be divided into two categories: the reader: the fixed the reader is suitable for data acquisition and processing and so on; the reader: This is suitable for mobile target tracking, positioning and so on. In the classification of the use of the words and can be divided into general oriented and mobile application oriented. Mainly in the use of RFID middleware is different from the professional, low cost, easy to develop, but not strong, and the high cost of mobile application development scale and targeted. This is also the research of several classification research in theory, no matter what kind of classification of the frame of the model is basically designed in hierarchical model, in the design of RFID middleware framework model has three functions is not the lack of hardware: equipment management, data processing and transmission, to provide services. These three functions of hierarchical cooperation constitute the basic framework of the RFID middleware, which is the core of data processing and transmission.

\section{RFID Middleware}

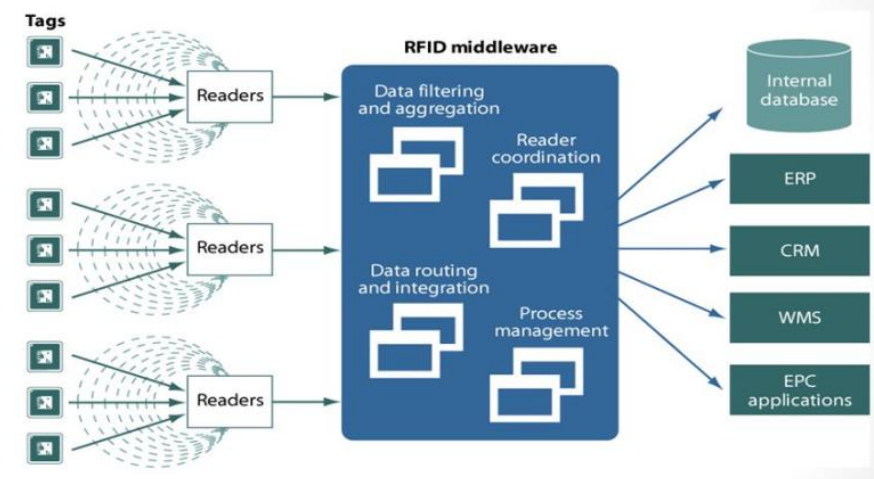

Figure 2. RFID Middleware

If according to the division of the module, then in the RFID middleware mainly has 5 big modules, the first is the application and hardware layer on both sides of the interface, that is, the business system interface module and RFID reader interface module. The middle of the data processing and equipment management module, that is, the logical 
reader mapping module, RFID data filtering module and equipment management module. The logical reader module is mapping the original data into the label business system at the beginning of data processing; RFID data filtering module is to read and write a lot of redundant tag data is filtered to get all available data, because there is no available data, duplicate data, error data in the original data and these data are not useful for business systems, must be processed by these modules.

\subsection{Load Balancing}

The load balancing mechanism is based on increasing the speed of processing the data of many servers, and the design of this mechanism should be stable. That is to say, the resources to be consumed in the system to provide load balancing algorithm mechanism components within the tolerance range, then this algorithm is not a good algorithm, need to improve or re design. The algorithm is good or bad is measured by the scale of the balance, in addition to the load balance is also based on the specific system structure design of different load balancing algorithm. In the general case, a simple load balancing LBA is used, the first to use the load of each server and the average value of the difference and the wait and wait:

$$
\begin{gathered}
A A L=\left(v_{a l_{01}}+v a l_{02}+\cdots+v a l_{n}\right) / n \\
L B A=\sum_{i=1}^{n}\left|A A L-v a l_{i}\right|
\end{gathered}
$$

AAL (The average amount of load) is the average value of the data volume to be processed on the $n$ server. When the load on each server is very average, that is, a high degree of load balance, then the load balance of LBA is very low. So at this time of the load balance degree LBA is LBdr divided by the total amount of $\mathrm{n}$ server.

$$
L B d r=L B A /(A A L \times n)
$$

So when the value of LBdr is lower, then the load balance is higher, which shows that the load at this time is very good. The following is a load balancing measure of memory, assuming that each server is input data from a unified load equalizer, and is at a certain point in the load situation.

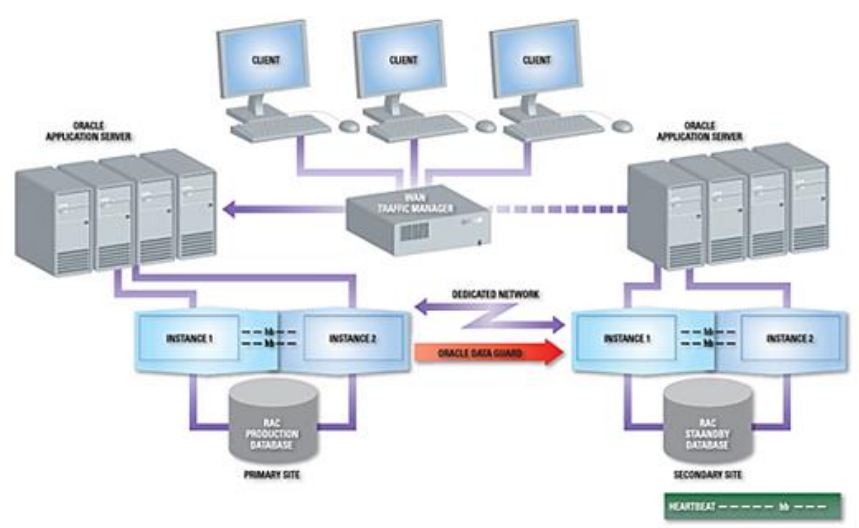

Figure 3. Server Processing Data Distribution

At this time the LBA is expressed as:

$$
\begin{gathered}
L B A=\left(\sum_{i=1}^{n}\left(\text { Smen }_{i}-\text { Tmen }_{i} / \text { Smen }_{i}\right)\right) \times K \times(1 / n) \\
L B d r=\left(\sum_{i=1}^{n}\left(\text { LBA }^{2}-\left(\text { Tmen }_{i} / \text { Smen }_{i}\right) \times K\right)^{2}\right) \times 100 \%
\end{gathered}
$$

Load balancing is a event handling ability of the state, this state can be divided into load equilibrium under static and dynamic conditions, the above measure is under static 
and dynamic conditions, basic and dynamic load of each server, and in the dynamic difference in each time interval after a time of. If the state of the server is non load balancing, then by the scheduling algorithm for scheduling, and this discovery is not balanced by scheduling to achieve scheduling algorithm for equilibrium strategies are triggered by the balance of the critical value of the load balancing to perform, when the load balance is higher than the critical value will pass the scheduling algorithm to make load balance the value is lower than the critical value. We call the load balancing algorithm including this scheduling algorithm, there is a more common is the beginning of the implementation of load balancing algorithm until the system is shut down, this algorithm and the scheduling algorithm have their advantages and disadvantages.

\section{Analysis of RFID Middleware System}

\subsection{Load Balancing Mechanism of RFID System}

RFID middleware system has two kinds of data communication format calculation: cloud computing and grid computing. There are two problems of load balancing. This section is mainly research on load balancing of grid middleware of RFID calculation, and the detailed study of cloud computing is mainly reflected in the sharing of data resources, but in grid computing middleware sometimes cloud computing is easy and fast, which is mainly used in the system and the communication system of single. The proposed grid based load balancing architecture is shown in Figure 4. There are more than one host for different application middleware. Each host is connected to a different number of read and write devices, and all are connected to the EPCIS through a grid platform. In addition, each host has two components, buffer management and load balancing management, in which the RFID middleware application provides load balancing mechanism.

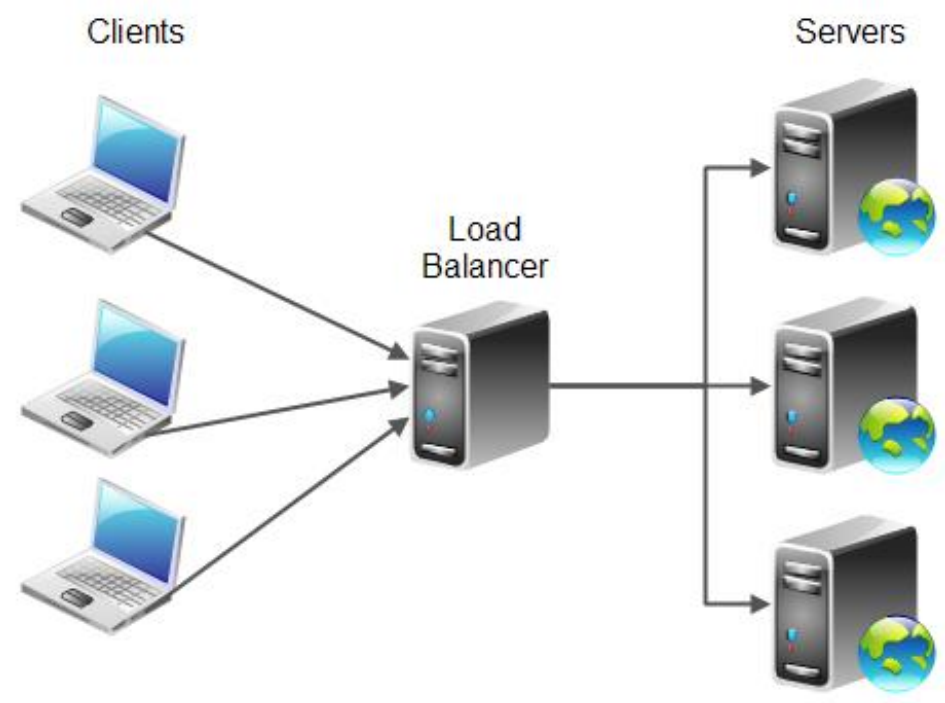

Figure 4. Load Balancing

Load balancing management and buffer management are designed in the middle of the two components. The following is a description of the two parts; as follows,

1) Buffer management: the buffer management module is the first communication connection between the reader and the reader. The tag data is read by the reader, and then transferred to the middleware, and then the buffer buffer management module is processed. Middleware will constantly check the buffer management module to ensure 
that all of the labeled data are processed. In addition to the tag buffer data, buffer management in the middleware host also records two thresholds to check whether the data buffer management is overloaded. User controls two thresholds so as to control the sensitive load balancing mechanism.

2) Load balancing management: load balance management is the core component of the load balancing mechanism. Load balancing management is based on the middleware, which is used to handle the load balancing problem of buffer management and middleware. It has four components, namely, a priority agent, a job locator, monitor, and a load balancing trigger. These components ensure that the load balancing mechanism is properly implemented.

- $\quad$ Priority agent: the priority agent component calculates the carrying capacity of each local middleware host, and sends the information back to the host of the middleware. In order to improve the computational efficiency, the mathematical model established in the priority agent is designed to be as simple as possible.

- Homework: This is also called job transfer locator; when the work location on the grid platform needs to be processed at the same time need to obtain the load capacity of each host, operation locator choose a lighter load operation in the middleware host group host. Job Locator records the load capacity of all middleware hosts and finds the lightest one by comparison. An overloaded host sends a message to the other middleware to indicate the status of the host. The job locator receives and records the overload message.

- Monitor: monitor the component to check the buffer data value in the buffer management area. If the amount of data reaches the maximum threshold of the set buffer management, the trigger start load balancing mechanism is monitored for the load balancing of the request.

- $\quad$ Load balancing trigger: load balancing trigger is the core component of the load balance management, when the load balancing mechanism to start the processing job transfers. As the core component of the load balance management, the trigger of load balancing in load balancing management needs to communicate with other components.

\subsection{Load Balancing Based on Agent Mode}

RLBA is designed to autonomously determine when and where to re allocate overloaded read and write devices, and to perform the re allocation of work. RFID middleware for RLBA to achieve the main functions of load balancing and implementation of the four strategies, starting strategy, job transfer strategy, selection strategy and positioning strategy. In the next section, the four strategies will be analyzed in detail. According to the definition of RFID middleware node standard RLBA implementation of a variety of agent load balancing strategy. RLBA is composed of five parts: the load monitor, the balanced trigger, the middleware selector, the reader to re distributor, the selector of the reader.

Load monitor: to determine which middleware to start load balancing mechanism, this middleware has the largest load of RFID middleware, or load the lightest one, or two. It also periodically checks to monitor the load of RFID middleware and periodically update RLT (Load Table Reader) on the local host. In fact, the load monitor is the starting strategy.

- Balanced trigger: deciding when to perform a node load balancing mechanism based on predefined criteria. At the sending end start condition, once the load is larger than the maximum load threshold, the load balancer will trigger this mentioned with us in the principle of load balancing mechanism as well as in the balance of trigger here and mentioned in the previous section load balancing is the same kind of trigger mechanism. Not to mention here.

- Middleware selector: this component is to determine which target RFID middleware to be used as a re distribution of the reader. In order to re assign and access 
GLT (Load Table Global) to determine the lightest load of RFID middleware. At the start of the receiver, the purpose of the middleware is the current RFID middleware.

Reader re distributor: this component is the reader to re distribution to the purpose of the middleware. Once the reader is assigned and determines which middleware is used as the destination of the middleware, the reader re distributor will be assigned to the selected reader. RFID reader manufacturers in order to facilitate the reader to re distributor development will also be developed to define a number of development kits.

- Reader selector: this component is to determine which reader is redistributed, reader selector visit RLT to select the load heavier or most of the reader to be assigned to the purpose of middleware, RFID middleware load until the local maximum threshold value is lower than the standard node load so far.

\section{Performance Test of Simulated Load Balancing}

\subsection{Simulated Load Balancing}

In the test load balancing performance before the completion of the first test load balancing simulation system, according to the test and input data items, the design of the simulation system can meet the performance of each algorithm test. The following is a brief exposition of the implementation of the simulation system. In the laboratory we have a blade server to provide this simulation system to test the use, there are a number of PC machines. A plurality of virtual machines can be installed on the blade server, and a plurality of operating systems can be installed on each virtual machine, and the simulation experiment is simulated on each operating system to simulate 1 middleware components. In this case, each virtual machine can simulate multiple RFID middleware components. And each PC machine can also be installed on a virtual machine, each virtual machine can also be loaded with 3 to 2 operating systems, each operating system can simulate 1 middleware components. In simulation experiments, we want to simulate the process of tag data into the middleware component, and the number of tag data and middleware components to change according to the requirements of the test. And set some parameters for each program or algorithm debugging. In most cases, a label at the same time will be two or more than two of the reader is detected, it is the heavy load of read and write tag data on re assigned to simultaneously detect another reader, then each reader is fixedly connected with RFID when a middleware, RFID middleware on all the reader are overburdened, will put the connection in the RFID middleware reader detection of label transfer to other RFID middleware, this algorithm can show the following diagram.

Set the Threshold value TVal $=N T / N R+N R / 2$;

For

\{

Find the reader tag data near to NOTV;

For

\{

Query a tag in the time stamp timestamp;

If (timestamp $==$ NULL)

Then Create a directory or entry in the memory of the tag, which is used to store the NOTV, ID, and time stamp of the reader.

Else if

Then $L V=$ The load value of the label

If ( LV > TVal \&\&NOTV<Tval)

Then Update the tab in the memory with the NOTV and ID of the current reader, and update the time stamp;

Else 


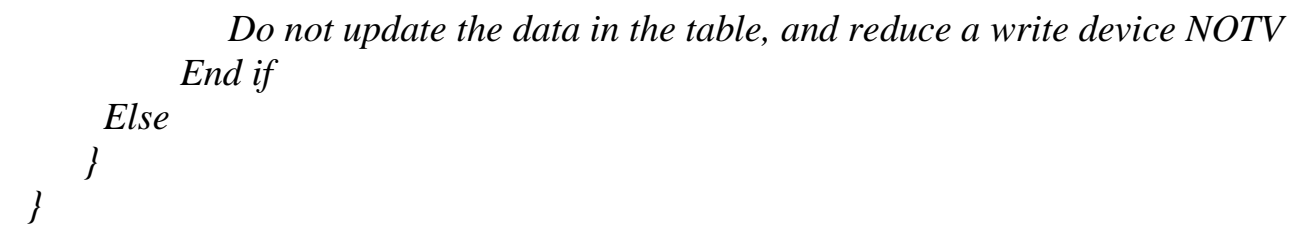

\subsection{Performance Testing and Comparative Analysis}

In the test some variables can be set, so that one or two variables can be fixed and the other one or two variables to see the load or operation of each program algorithm. The simulation experiment is the number of tags and the number of middleware to open the number of these two parameters set to the amount of intervention. It is better to set the number of tags as quantitative, the number of middleware is set as a variable, according to the number of different purposes of the middleware to test the load of different algorithms.

The first test is the consumption of energy and load balance. For testing the consumption of energy is a dynamic number of open read and write devices, so the number of open reader is dynamic, and the number of the middleware is fixed, we set the number of middleware to open MW_num=30. Thus we test the optimal scheme of reading labels, RRE (eliminate redundant reader Elimination Redundant), agent, distributed ALE way, the test of the four algorithms are as follows:

1) Load balancing rate: the test load balancing rate is used to calculate the load balance of the different types of programs or algorithms.

2) The missing rate of the label: available Rate tag= (the total amount of tags - has been processed in the system Num) / (the total amount of TNUM) is to calculate the rate of loss. The tag that has been entered in the system is not filtered but has already entered the tag of the reader.

3) The throughput rate: Throughput= (the number of tags in a period of time Num) / (T); that is, the number of tags in a unit of time processing. Throughput is the number of tags that are processed within the specified time.

4) The consumption of energy: which refers to the consumption of energy refers to the dynamic of the number of read and write devices. The number of the reader to simulate the energy consumption, when the dynamic open reader with more words, the energy consumption is large, and vice versa.

5) The average processing time: $\mathrm{APT}=$ (the time required for the middleware to handle the total number of tags (T) / (a number of tags of num). In addition to the above test scalar quantity, there are also a number of tests in our consideration. Such as response time, that is, from the beginning of the tag data processing to deal with the end of the time; and then the memory of each server.

In the test some variables can be set, so that one or two variables can be fixed and the other one or two variables to see the load or operation of each program algorithm. The simulation experiment is the number of tags and the number of middleware to open the number of these two parameters set to the amount of intervention. It is better to set the number of tags as quantitative, the number of middleware is set as a variable, according to the number of different purposes of the middleware to test the load of different algorithms. The first test is the consumption of energy and load balance. For testing the consumption of energy is a dynamic number of open read and write devices, so the number of open reader is dynamic, and the number of the middleware is fixed, we set the number of middleware to open MW_num=30. Thus we test the optimal scheme of reading labels, RRE (eliminate redundant reader Elimination Redundant), agent, distributed ALE way, the test of the four algorithms are as follows: 


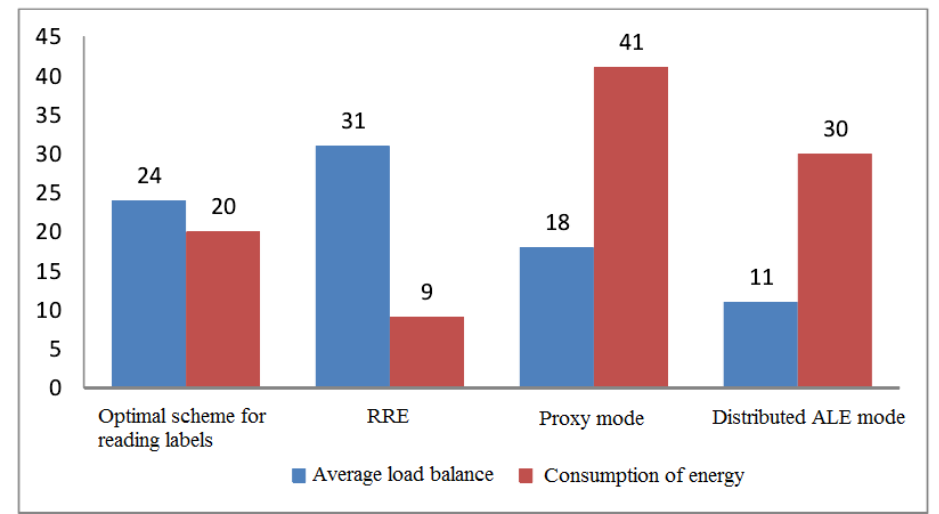

\section{Figure 5. Comparison of Energy Dissipation and Load Balancing}

According to the above load balancing test average LBdr, you can draw a line chart, to compare the four algorithms intuitive good or bad. As shown in Figure four is the 6 kinds of load balancing algorithm in the number of tags.

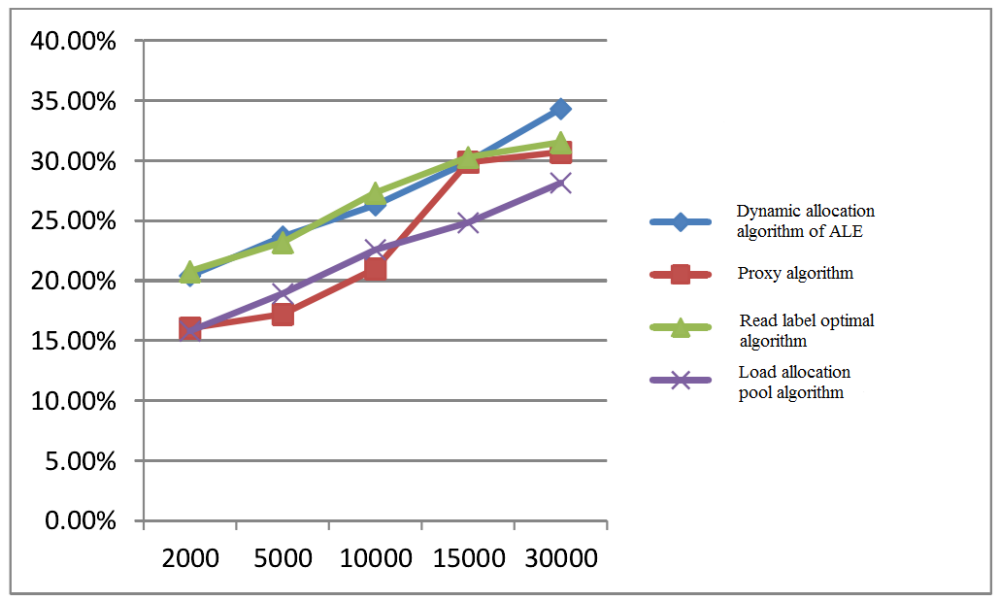

Figure 6. Load Balancing Algorithm

It can be seen in Figure 6, in which four kinds of algorithm in load allocation pool algorithm is a better algorithm, but when the number of tags in which the agent algorithm is relatively small, load balance degree is relatively low, but when the number of tags to balance and read the label 15000 time optimal algorithm is almost the same, then it is not good algorithm. We can also see the four algorithms of LBdr are basically in $15 \%$ above, if in the actual production in order to pursue the load, the load distribution can choose pool algorithm, because this algorithm is the best of the four. But there is also a deviation, because this test is the number of read and writes devices and middleware is set very low, but in the actual number of producers and the number of the reader writer and middleware will be a lot of. So there may be some bias in the test and practice.

For a good system, will require that the system has a very high data throughput rate, the next is to test the tag data of four algorithms throughput test, we still have to fix some variables, the fixed number of Middleware in 20, the number of tags in the 100020003000500010000 series of four stimulation test algorithm the rate of simulation experiment is to see has to deal with the number of labels num in half a minute, in the use of num $/ 30$ s, then can get the unit 1 seconds has been processed, the number of labels. In order to facilitate the calculation of the time fixed to 30 seconds, that is, $\mathrm{T}=30$. The throughput rate of the unit is (one per second) shown in the following table is the result of the test calculation table: 
Table 2. Table of Calculation Results of Throughput

\begin{tabular}{|c|c|c|c|c|}
\hline Algorithm type & $\begin{array}{c}\text { Dynamic ALE } \\
\text { allocation } \\
\text { algorithm }\end{array}$ & $\begin{array}{c}\text { Proxy } \\
\text { algorithm }\end{array}$ & $\begin{array}{c}\text { Read label } \\
\text { optimal } \\
\text { algorithm }\end{array}$ & $\begin{array}{c}\text { Load } \\
\text { distribution } \\
\text { pool } \\
\text { algorithm }\end{array}$ \\
\hline 1000 & 46 & 33 & 49 & 39 \\
\hline 2000 & 76 & 79 & 54 & 62 \\
\hline 3000 & 96 & 99 & 76 & 76 \\
\hline 5000 & 126 & 97 & 95 & 93 \\
\hline 10000 & 133 & 100 & 112 & 102 \\
\hline
\end{tabular}

As shown in Figure 7, the data on this figure is also the average number of 3 tests. As can be seen from Figure 7, the throughput rate of the agent algorithm is 3000 when the number of tags, and then to the back of the throughput rate has been stable. Better throughput is a dynamic allocation algorithm for ALE. While the other throughput rate is increasing with the number of tags, the rate of the increase of the throughput rate is not very large.

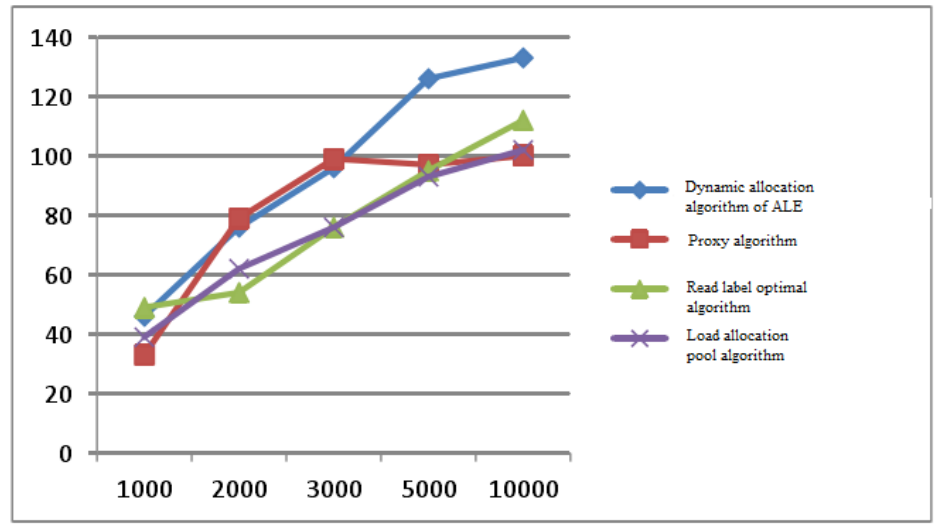

Figure 7. Test of the Rate of the Label of the Algorithm

In this experiment, we also tested the average processing time in the middle of the input to the application layer after the tag was processed in the middle of the input to the middle of the application layer after the reader's output was read out. In the average processing time of the test label, first to set the number of middleware to open, the number of the middleware to open here is 40 . Test change is the number of tags, the reader reads the tag, the tag data into the middleware system in the number of values. The algorithm is tested by simulation software test first by experiment, and then three times the test value using the formula to calculate the average processing time of three times, three times and then averaged to arrive at a final show in the figure data. 


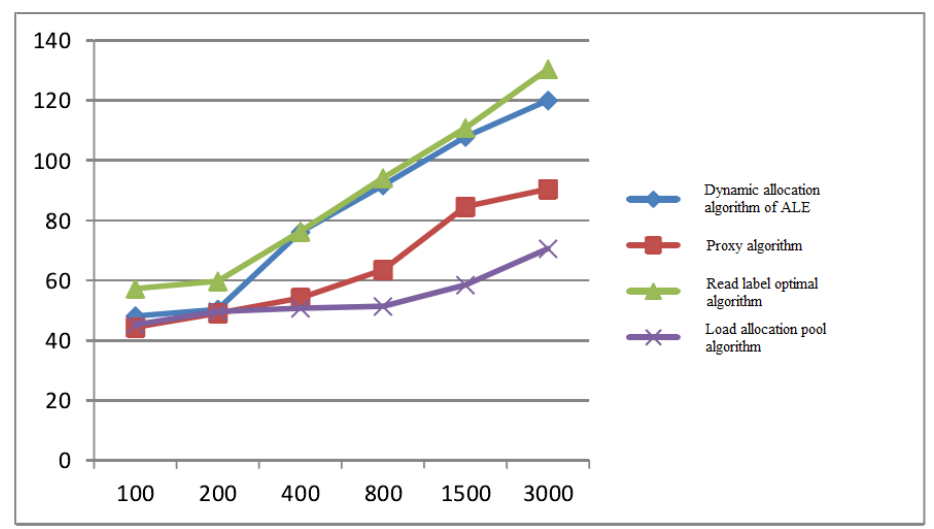

Figure 8. Average Processing Time of the Algorithm

It can be seen that in an order of magnitude of the label, the four the time required by the algorithm is increased with the increase of the number of labels, but the processing time of the load distribution of pool less than the other three algorithms of processing time. The results obtained from this experiment are basically consistent with our previous predictions, but there are some deviations in some aspects. This is mainly the error in the experiment.

\section{Conclusions}

This paper is mainly based on the research of RFID technology, especially the RFID based Middleware Technology, and the RFID middleware framework, the relationship between the various components of the middleware, and processing the tag data for the detailed research and analysis. In addition, the load balancing algorithm on some of their views and research, so the load of middleware, and analysis to design their own new architecture in RFID load balancing middleware and the algorithm through the load balancing algorithm of combination. In the meantime, by reading the RFID middleware technology and the foreign data and based on the research on the application of RFID technology on the proposed own RFID middleware architecture and load balancing algorithm, and test the ratio analysis to put forward their own algorithm and already existing algorithms, I proposed algorithm is superior to the existing RFID Middleware architecture and load balancing algorithm in processing and labeling on the average time.

\section{Acknowledgments}

This research was supported by Zhejiang Province public nonprofit technology applied research plan project(No. 2015C33236), Zhejiang Province University youth discipline leader academic climbing program(No. pd2013443), Ningbo Natural Science Foundation project(No.2012A610071).

\section{References}

[1] G. Huang and H. Li, "RFID-enabled real-time PBS monitoring for automobile assembly factory", International Journal of Computer Integrated Manufacturing, vol. 25, (2012), pp. 66-85.

[2] C. Saygin and S. Tamma, "RFID-enabled shared resource management for aerospace maintenance operations:a dynamic resource allocation model", International Journal of Computer Integrated Manufacturing, vol. 25, (2012), pp. 100-111.

[3] M. Lianbo and H. Hu, "The development of RFID middleware based on multi-level data processing", Computer Engineering, vol. 39, (2010), pp. 289-297.

[4] Z. Huang and M. Benyoucef, "Consistenthashing dynamic feedback load balancing algorithm", microelectronics and computer, vol. 12, no. 4, (2013), pp. 246-259.

[5] C. Jing, "Research on the management and coordination of RFID middleware reader management and coordination", computer applications and software, vol. 37, (2012), pp. 64-67. 
[6] W. Quanyuan, "Research on distributed high scalable load balancing middleware technology", Computer Engineering, vol. 33, (2012), pp. 1144-1148.

[7] Z. Jinxia, "The architecture and implementation of distributed multi protocol", computer engineering and design, vol. 18, (2015), pp. 381-387.

[8] L. Zilu, "Load balancing technology of shunt based distributed packet switching parallel", Computer research and development, vol. 40, no. 2, (2014), pp. 152-162.

[9] W. Hongwei, "Design and implementation of real time event processing mechanism for RFID middleware", logistics technology, vol. 17, (2012), pp. 1897-1900. 